\title{
A Remark on the Global Attractors of the Nonlinear Evolution Equations
}

\author{
Chang Ya-ya and Ma Qiao-zhen \\ School of Mathematics and Statistics, Northwest Normal University, Lanzhou, Gansu 730070, China \\ Correspondence should be addressed to Ma Qiao-zhen; maqzh@nwnu.edu.cn \\ Received 18 September 2013; Accepted 13 November 2013 \\ Academic Editors: H. Homeier and J. Kou
}

Copyright (c) 2013 C. Ya-ya and M. Qiao-zhen. This is an open access article distributed under the Creative Commons Attribution License, which permits unrestricted use, distribution, and reproduction in any medium, provided the original work is properly cited.

We study the existence of global attractor of the nonlinear elastic rod oscillation equation when the forcing term belongs only to $H^{-1}(\Omega)$; furthermore, we prove that the fractal dimension of global attractor is finite.

\section{Introduction}

Let $\Omega$ be an open bounded set of $\mathbb{R}^{3}$ with smooth boundary $\partial \Omega$. We consider the following equation:

$$
\begin{array}{r}
u_{t t}-\Delta u-\Delta u_{t}-\omega \Delta u_{t t}+f(u)=g(x), \\
(x, t) \in \Omega \times \mathbb{R}^{+}, \\
\left.u\right|_{t=0}=u_{0},\left.\quad u_{t}\right|_{t=0}=u_{1}, \quad \forall x \in \Omega, \\
\left.u\right|_{\partial \Omega}=0, \quad \forall t \geq 0,
\end{array}
$$

where $\omega>0$ and $g \in H^{-1}(\Omega)$. The nonlinear term $f \in$ $C^{1}(\mathbb{R}, \mathbb{R}), f(0)=0$, and satisfies the following:

$$
\begin{gathered}
\liminf _{|s| \rightarrow \infty} \frac{f(s)}{s}>-\lambda_{1}, \\
\left|f^{\prime}(s)\right| \leqslant C\left(1+|s|^{4}\right), \quad \forall s \in \mathbb{R},
\end{gathered}
$$

where $\lambda_{1}$ is the first eigenvalue of $-\Delta$ in $H_{0}^{1}(\Omega)$ and $C$ is a positive constant.

In line with the Galerkin methods introduced in [1], we know that (1) has a unique solution $u \in C\left([0, T] ; H_{0}^{1}(\Omega)\right)$, $u_{t} \in$ $C\left([0, T] ; H_{0}^{1}(\Omega)\right)$, for $g \in H^{-1}(\Omega)$. The proof has no essential difference between $g(x) \in L^{2}(\Omega)$ and $g(x) \in H^{-1}(\Omega)$, so we omit it; see [2].

Equation (1), which appears as a class of nonlinear evolution equations, like the strain solitary wave equation and dispersive-dissipative wave equation, is used to represent the propagation problems of a lengthwise wave in nonlinear elastic rods and lon-sonic of space transformation by weak nonlinear effect; see [3-6]. For (1), when $g(x) \in L^{2}(\Omega)$, in [2], the author has discussed the existence of global strong solutions in $\left(H^{2}(\Omega) \cap H_{0}^{1}(\Omega)\right) \times\left(H^{2}(\Omega) \cap H_{0}^{1}(\Omega)\right)$; in [7, 8], the authors have obtained the existence of global attractors in the weak topological space and the strong topology space, respectively. Recently, existence of the uniform compact attractors has been proved about the nonautonomous case of (1); that is, $g(x)=g(x, t)$. In this paper, we prove existence of global attractor and its fractal dimension for (1) under the condition that $g(x)$ only satisfies the lower regularity.

\section{The Main Results}

Without loss of generality, we denote $H=L^{2}(\Omega), V=H_{0}^{1}(\Omega)$, and $H^{*}, V^{*}$ is, respectively, the dual space of $H, V$. Write $\mathscr{H}_{1}=H_{0}^{1}(\Omega) \times H_{0}^{1}(\Omega)$. Let $A=-\Delta$ and $D(A)=H^{2}(\Omega) \cap$ $H_{0}^{1}(\Omega)$; we define $D\left(A^{(s / 2)}\right) ; s \in \mathbb{R}$ is Hilbert space family, and its inner product and norm are

$$
(\cdot, \cdot)_{D\left(A^{(s / 2)}\right)}=\left(A^{(s / 2)} \cdot A^{(s / 2)} \cdot\right), \quad\|\cdot\|_{D\left(A^{(s / 2)}\right)}=\left\|A^{(s / 2)} \cdot\right\| .
$$

The following results will be used later.

Lemma 1 (see [8]). Assume that $f$ satisfies (2) and (3), $g \in$ $H^{-1}(\Omega)$; then, the solution semigroup $\{S(t)\}_{t \geq 0}$ has a bounded 
absorbing set $\mathscr{B}_{0}$ in $\mathscr{H}_{1}$; that is, for any bounded subset $\mathscr{B} \subset$ $\mathscr{H}_{1}$, there exists $T=T(\mathscr{B})$ such that

$$
S(t) \mathscr{B} \subset \mathscr{B}_{0}, \quad \forall t \geq T
$$

Lemma 2. Let $\Omega \subset \mathbb{R}^{3}$ be a bounded domain with smooth boundary, and one assumes that $f$ satisfies (2) and (3), $g \in H^{-1}(\Omega)$; then, the semigroup $\{S(t)\}_{t \geq 0}$ possesses a global attractor $\mathscr{A}_{0}$ on $\mathscr{H}_{1}$.

Proof. Since $L^{2}(\Omega) \hookrightarrow H^{-1}(\Omega)$ is dense, for any $\eta>0$, there exists $g(x) \in L^{2}(\Omega)$ such that

$$
\left\|g(x)-g^{\eta}(x)\right\|_{H^{-1}} \leq \eta, \quad \text { for any } g \in H^{-1}(\Omega) .
$$

The remained proof of Lemma 2 is similar to that of [7], so we omit it.

Lemma 3 (see [9]). Let $B$ be a bounded subset in Hilbert space $X$, the mapping $V: B \rightarrow X$, such that $B \subseteq V(B)$, and satisfy

$$
\begin{gathered}
\|V(v)-V(\widetilde{v})\|_{X} \leq l\|v-\widetilde{v}\|_{X}, \quad \forall v, \widetilde{v} \in B, \\
\left\|Q_{N} V(v)-Q_{N} V(\widetilde{v})\right\|_{X} \leq \delta\|v-\widetilde{v}\|_{X}, \quad(0<\delta<1),
\end{gathered}
$$

where $Q_{N}: X \rightarrow X_{N}^{\perp}$ is orthogonal mapping and $X_{N}$ is spanned subspace by the former $N$ th eigenvector of $X$; then, the fractal dimension of $B$ satisfies

$$
d_{F}(B) \leq \frac{N \ln \left(8 k^{2} l^{2} /\left(1-\delta^{2}\right)\right)}{\ln \left(2 /\left(1-\delta^{2}\right)\right)},
$$

where $k$ is the Gaussian constant.

Our main result is as follows.

Theorem 4. Let $\Omega \subset \mathbb{R}^{3}$ be a bounded domain with smooth boundary; one assumes that $f$ satisfies (2) and (3), $g \in H^{-1}(\Omega)$; then, the fractal dimension of the global attractor $\mathscr{A}_{0}$ of the semigroup $\{S(t)\}_{t \geq 0}$ is finite.

Proof. According to Lemma 2, provided

$$
u(x, 0)=u_{0}(x), \quad v(x, 0)=v_{0}(x) \in \mathscr{A}_{0} ;
$$

then,

$$
u(x, t)=S(t) u_{0}, \quad v(x, t)=S(t) v_{0} \in \mathscr{A}_{0} .
$$

Let $w=u-v$ satisfy the following equation:

$$
w_{t t}-\Delta w-\Delta w_{t}-\omega \Delta w_{t t}+f(u)-f(v)=0
$$

Taking the scalar product of $(11)$ in $L^{2}(\Omega)$ with $w_{t}$, we obtain that

$$
\begin{aligned}
\frac{1}{2} \frac{d}{d t}( & \left.\left\|w_{t}\right\|^{2}+\left\|A^{(1 / 2)} w\right\|^{2}+\omega\left\|A^{(1 / 2)} w_{t}\right\|^{2}\right) \\
& +\left\|A^{(1 / 2)} w_{t}\right\|^{2}+\left(f(u)-f(v), w_{t}\right)=0 .
\end{aligned}
$$

As the global attractor $\mathscr{A}_{0}$ is bounded in $\mathscr{H}_{1}(\Omega)$, so there exists $M_{0}>0$ such that

$\max _{x \in \Omega}\|u\|, \quad \max _{x \in \Omega}\|v\|, \quad\left\|A^{(1 / 2)} u\right\|, \quad\left\|A^{(1 / 2)} v\right\| \leq M_{0}$.

Therefore, using (3) and (13), it follows that

$$
\begin{aligned}
\left(f(u)-f(v), w_{t}\right) & =\left|\int_{0}^{1}\left(f^{\prime}(\theta u+(1-\theta) v) d \theta \cdot w, w_{t}\right)\right| \\
& \leq \int_{\Omega}\left|\int_{0}^{1} f^{\prime}(\theta u+(1-\theta) v) d \theta\right| \\
& \cdot|w| \cdot\left|w_{t}\right| d x \\
& \leq C_{1} \int_{\Omega}\left(1+|u|^{4}+|v|^{4}\right) \cdot|w| \cdot\left|w_{t}\right| d x \\
& \leq C_{2} \int_{\Omega}|w|\left|w_{t}\right| d x \\
& \leq \frac{C_{2}}{2 \lambda_{1}}\left\|A^{(1 / 2)} w\right\|^{2}+\frac{C_{2}}{2}\left\|w_{t}\right\|^{2}
\end{aligned}
$$

By (12) and (14), we obtain

$$
\begin{aligned}
& \frac{d}{d t}\left(\left\|w_{t}\right\|^{2}+\left\|A^{(1 / 2)} w\right\|^{2}+\omega\left\|A^{(1 / 2)} w_{t}\right\|^{2}\right) \\
& \quad \leq 2 C_{3}\left(\left\|w_{t}\right\|^{2}+\left\|A^{(1 / 2)} w\right\|^{2}+\left\|A^{(1 / 2)} w_{t}\right\|^{2}\right)
\end{aligned}
$$

where $C_{i}(i=1,2,3)$ is constant independent of $\omega$; by Gronwall's inequality, we get

$$
\begin{aligned}
\left\|w_{t}\right\|^{2} & +\left\|A^{(1 / 2)} w\right\|^{2}+\omega\left\|A^{(1 / 2)} w_{t}\right\|^{2} \\
& \leq\left(\left\|w_{t}(0)\right\|^{2}+\left\|A^{(1 / 2)} w(0)\right\|^{2}+\left\|A^{(1 / 2)} w_{t}(0)\right\|^{2}\right) e^{2 C_{3} t} .
\end{aligned}
$$

For some $t_{1}>0$, define $l=e^{2 C_{3} t_{1}}$; hence, we prove that the first inequality in Lemma 3 holds true.

Taking the inner product of (11) in $L^{2}(\Omega)$ with $Q_{N} w_{t}(x, t)$, we commute the operator $A$ with the projection $Q_{N}$ to get

$$
\begin{aligned}
\frac{1}{2} \frac{d}{d t}( & \left.\left\|Q_{N} w_{t}\right\|^{2}+\left\|A^{(1 / 2)} Q_{N} w\right\|^{2}+\omega\left\|A^{(1 / 2)} Q_{N} w_{t}\right\|^{2}\right) \\
& +\left\|A^{(1 / 2)} Q_{N} w_{t}\right\|^{2}+\left(f(u)-f(v), Q_{N} w_{t}\right)=0 .
\end{aligned}
$$

Similar to the estimates of (13)-(14), we obtain

$$
\begin{aligned}
& \int_{\Omega}\left|(f(u)-f(v)) \cdot Q_{N} w_{t}\right| d x \\
& \leq \int_{\Omega}\left|f^{\prime}(\theta u+(1-\theta) v)\right| \\
& \quad \cdot|w| \cdot\left|Q_{N} w_{t}\right| d x \leq C_{4}\|w\| \cdot\left\|Q_{N} w_{t}\right\|
\end{aligned}
$$




$$
\begin{aligned}
& \leq C_{4}\|w\| \cdot\left\|A^{(1 / 2)} Q_{N} w_{t}\right\| \lambda_{N+1}^{-(1 / 2)} \\
& \leq \frac{C_{4}}{2} \lambda_{N+1}^{-(1 / 2)}\|w\|^{2}+\frac{C_{4}}{2} \lambda_{N+1}^{-(1 / 2)}\left\|Q_{N} A^{(1 / 2)} w_{t}\right\|^{2} \\
& \leq \frac{C_{4}}{2 \lambda_{1}} \lambda_{N+1}^{-(1 / 2)}\left\|A^{(1 / 2)} w\right\|^{2}+\frac{C_{4}}{2} \lambda_{N+1}^{-(1 / 2)}\left\|Q_{N} A^{(1 / 2)} w_{t}\right\|^{2} .
\end{aligned}
$$

Then,

$$
\begin{aligned}
\frac{1}{2} \frac{d}{d t} & \left(\left\|Q_{N} w_{t}\right\|^{2}+\left\|A^{(1 / 2)} Q_{N} w\right\|^{2}+\omega\left\|A^{(1 / 2)} Q_{N} w_{t}\right\|^{2}\right) \\
& +\left\|A^{(1 / 2)} Q_{N} w_{t}\right\|^{2} \leq \frac{C_{4}}{2 \lambda_{1}} \lambda_{N+1}^{-(1 / 2)}\left\|A^{(1 / 2)} w\right\|^{2} \\
& +\frac{C_{4}}{2} \lambda_{N+1}^{-(1 / 2)}\left\|A^{(1 / 2)} Q_{N} w_{t}\right\|^{2}
\end{aligned}
$$

where $\lambda_{N}$ is the $N$ th eigenvalue of problem (11), so we have

$$
\begin{aligned}
\frac{d}{d t}( & \left.\left\|Q_{N} w_{t}\right\|^{2}+\left\|A^{(1 / 2)} Q_{N} w\right\|^{2}+\omega\left\|A^{(1 / 2)} Q_{N} w_{t}\right\|^{2}\right) \\
& +\lambda_{N+1}^{(1 / 2)}\left\|Q_{N} w_{t}\right\|^{2}+\lambda_{N+1}^{-1}\left\|A^{(1 / 2)} Q_{N} w\right\|^{2} \\
& +\left(1-C_{4} \lambda_{N+1}^{-(1 / 2)}\right)\left\|A^{(1 / 2)} Q_{N} w_{t}\right\|^{2} \\
\leq & \left(\frac{C_{4}}{\lambda_{1}} \lambda_{N+1}^{-(1 / 2)}+\lambda_{N+1}^{-1}\right)\left\|A^{(1 / 2)} w\right\|^{2} .
\end{aligned}
$$

We let

$$
y(t)=\left\|Q_{N} w_{t}\right\|^{2}+\left\|A^{(1 / 2)} Q_{N} w\right\|^{2}+\omega\left\|A^{(1 / 2)} Q_{N} w_{t}\right\|^{2} .
$$

Choosing $N$ large enough that $1-C_{4} \lambda_{N+1}^{-(1 / 2)}>0$ and setting $\alpha=\min \left\{\lambda_{N+1}^{-1}, \lambda_{N+1}^{(1 / 2)}, 1-C_{4} \lambda_{N+1}^{-(1 / 2)}\right\}$, integrating with (16) and (20), we get

$$
\begin{aligned}
y^{\prime}(t) & +\alpha y(t) \leq \frac{C_{4}}{\lambda_{1}} \lambda_{N+1}^{-(1 / 2)} \\
& \times\left(\left\|w_{t}(0)\right\|^{2}+\left\|A^{(1 / 2)} w(0)\right\|^{2}+\left\|A^{(1 / 2)} w_{t}(0)\right\|^{2}\right) e^{2 C_{3} t}
\end{aligned}
$$

Gronwall's inequality implies that

$$
\begin{aligned}
y(t) \leq & y(0) e^{-\alpha t}+\frac{1}{2 C_{3}+\alpha}\left(\frac{C_{4}}{\lambda_{1}} \lambda_{N+1}^{-(1 / 2)}+\lambda_{N+1}^{-1}\right) \\
& \times\left(\left\|w_{t}(0)\right\|^{2}+\left\|A^{(1 / 2)} w(0)\right\|^{2}+\left\|A^{(1 / 2)} w_{t}(0)\right\|^{2}\right) e^{2 C_{3} t} . \\
\leq & \left(\left\|w_{t}(0)\right\|^{2}+\left\|A^{(1 / 2)} w(0)\right\|^{2}+\left\|A^{(1 / 2)} w_{t}(0)\right\|^{2}\right) \\
& \times\left(e^{-\alpha t}+\frac{1}{2 C_{3}+\alpha}\left(\frac{C_{4}}{\lambda_{1}} \lambda_{N+1}^{-(1 / 2)}+\lambda_{N+1}^{-1}\right) e^{2 C_{3} t}\right) .
\end{aligned}
$$

So, we have

$$
\begin{aligned}
\left\|Q_{N} w\right\|_{\mathscr{H}_{1}}^{2} \leq & y(t) \leq\left\|w_{0}\right\|_{\mathscr{H}_{1}}^{2} \\
& \times\left(e^{-\alpha t}+\frac{1}{2 C_{3}+\alpha}\left(\frac{C_{4}}{\lambda_{1}} \lambda_{N+1}^{-(1 / 2)}+\lambda_{N+1}^{-1}\right) e^{2 C_{3} t}\right) .
\end{aligned}
$$

Take a proper $t_{1}>0$ and $N$ large enough such that

$$
e^{-\alpha t_{1}}+\frac{1}{2 C_{3}+\alpha}\left(\frac{C_{4}}{\lambda_{1}} \lambda_{N+1}^{-(1 / 2)}+\lambda_{N+1}^{-1}\right) e^{2 C_{3} t_{1}} \leq \delta<1 .
$$

Therefore, for $t=t_{1}, S\left(t_{1}\right)$ satisfies the condition of Lemma 3; then, the fractal dimension of the global attractor $\mathscr{A}_{0}$ satisfies

$$
d_{F}(B) \leq \frac{N \ln \left(8 k^{2} l^{2} /\left(1-\delta^{2}\right)\right)}{\ln \left(2 /\left(1-\delta^{2}\right)\right)} .
$$

This implies that the global attractor for semigroup $\{S(t)\}_{t \geq 0}$ generated by the problem (1) has a finite fractal dimension.

\section{Acknowledgments}

This work was partly supported by the NSFC (11101334) and the NSF of Gansu Province (1107RJZA223), and by the Fundamental Research Funds for the Gansu Universities.

\section{References}

[1] R. Temam, Infinite-Dimensional Dynamical Systems in Mechanics and Physics, vol. 68 of Applied Mathematical Sciences, Springer, New York, NY, USA, 2nd edition, 1997.

[2] Y. D. Shang, "Initial-boundary value problem for the equation $u_{t t}-\Delta u-\Delta u_{t}-\Delta u_{t t}=f(u), "$ Acta Mathematicae Applicatae Sinica, vol. 23, no. 3, pp. 385-393, 2000.

[3] I. L. Bogolubky, "Some examples of inelastic solution interaction," Computer Physics Communications, vol. 13, 1977.

[4] W. G. Zhu, "Nonlinear waves in elastic rods," Acta Solid Mechanica Sinica, vol. 1, no. 2, pp. 247-253, 1980.

[5] C. E. Seyler and D. L. Fanstermacher, "Asymmetric regularized long wave equation," Physics of Fluids, vol. 27, no. 1, pp. 58-66, 1984.

[6] P. A. Clarkson, R. J. LeVeque, and R. Saxton, "Solitary-wave interactions in elastic rods," Studies in Applied Mathematics, vol. 75, no. 2, pp. 95-121, 1986.

[7] Y. Xie and C. Zhong, "The existence of global attractors for a class nonlinear evolution equation," Journal of Mathematical Analysis and Applications, vol. 336, no. 1, pp. 54-69, 2007.

[8] Y. Xie and C. Zhong, "Asymptotic behavior of a class of nonlinear evolution equations," Nonlinear Analysis. Theory, Methods \& Applications, vol. 71, no. 11, pp. 5095-5105, 2009.

[9] A. O. Çelebi, V. K. Kalantarov, and M. Polat, "Attractors for the generalized Benjamin-Bona-Mahony equation," Journal of Differential Equations, vol. 157, no. 2, pp. 439-451, 1999. 


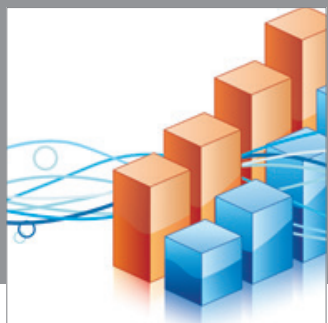

Advances in

Operations Research

mansans

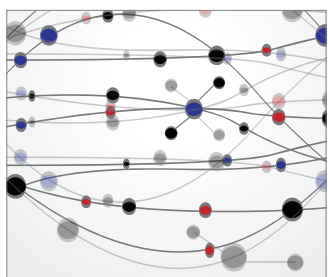

The Scientific World Journal
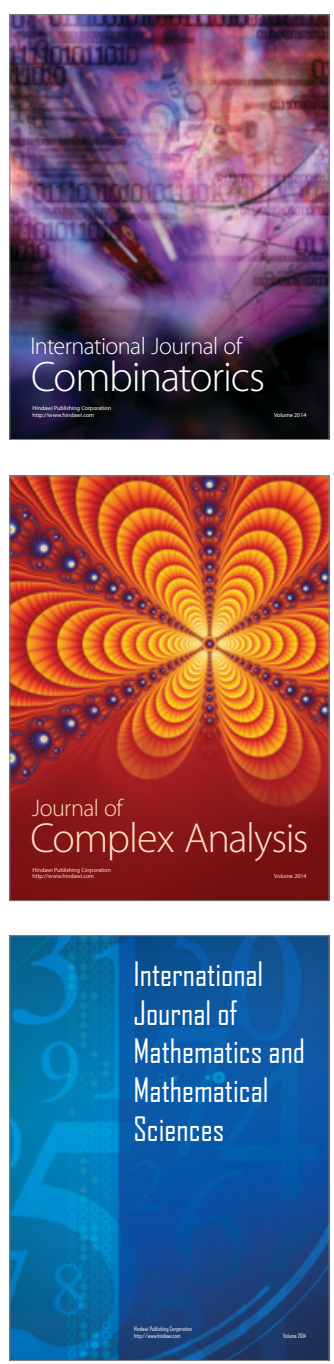
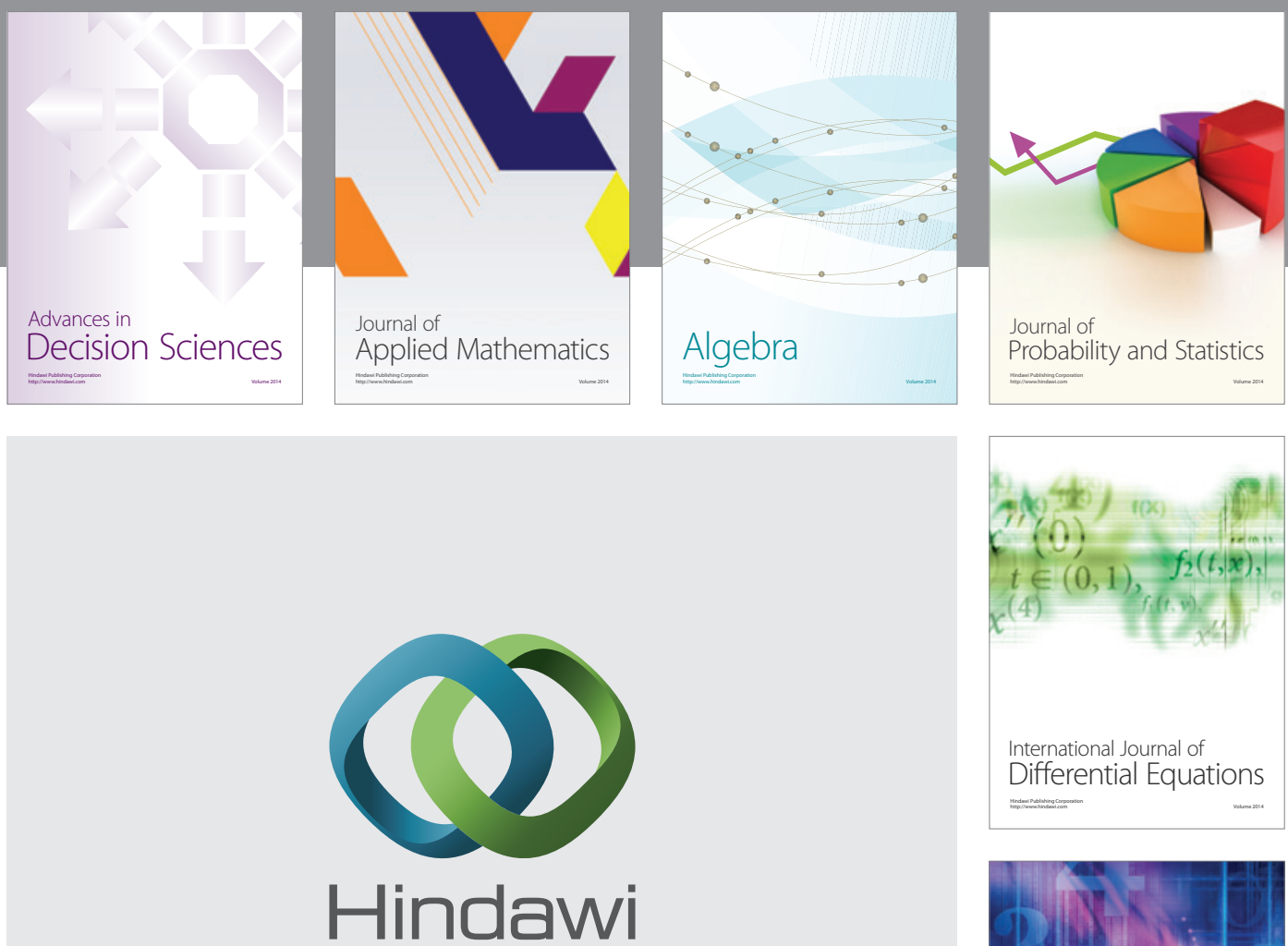

Submit your manuscripts at http://www.hindawi.com
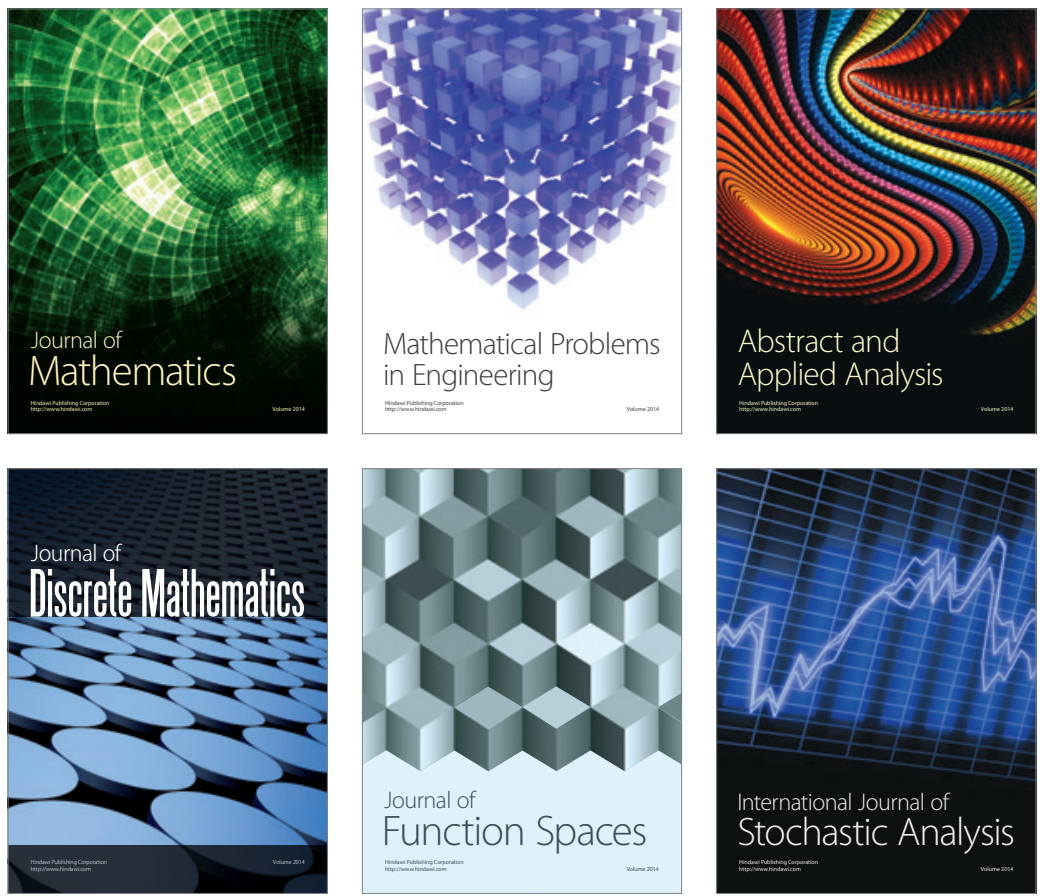

Journal of

Function Spaces

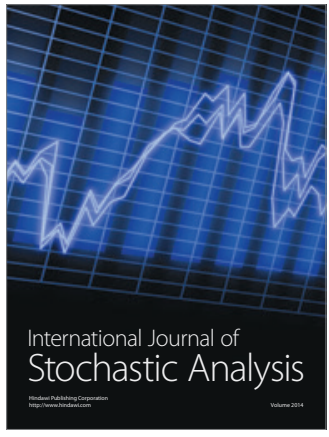

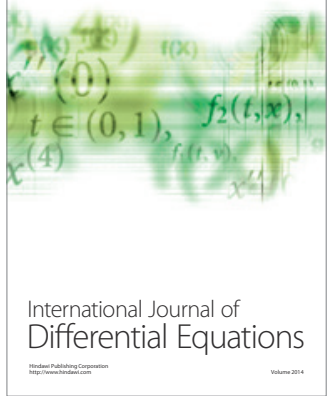
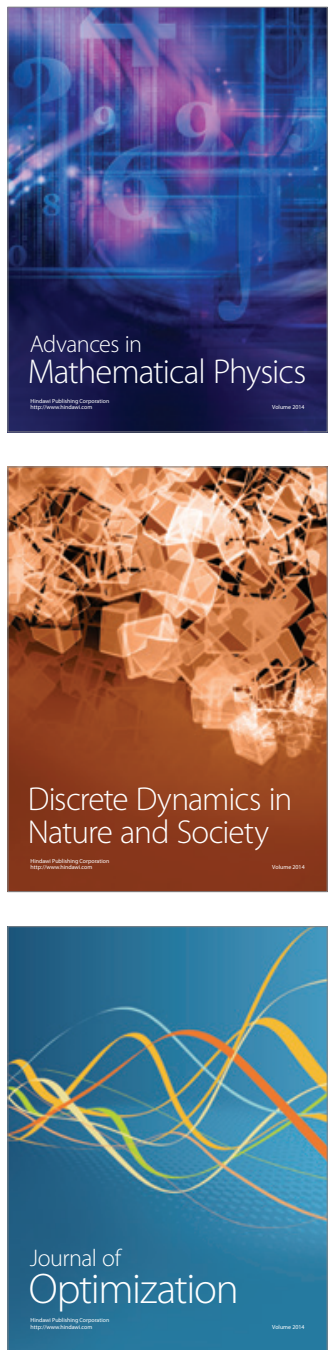